\title{
Evaluation of Immunogenicity, Protective Immunity on Aquaculture Pathogenic Vibrio and Fermentation of Vibrio alginolyticus Flagellin FlaC Protein
}

\author{
Chen Chen ", Chao Kang ${ }^{1}$, Na Rong ${ }^{1}$, Nana Wu ${ }^{1}$, Chunlin Chen ${ }^{~}$, Sanqiao Wu ${ }^{1}$, Xiaoying Zhang 1,2,*, \\ Xiang Liu ${ }^{1}{ }^{*}$ \\ ${ }^{1}$ Chinese-German joint Institute for natural product research / Shaanxi Engineering Research Center for Tall Gastrodia \\ Tuber and Medical Dogwood / College of Biological Science and Engineering, Shaanxi University of Technology, \\ Hanzhong, China \\ ${ }^{2}$ Centre of Molecular and Environmental Biology University of Minho, Department of Biology, Campus de Gualtar, Braga, \\ Portugal
} * Corresponding authors: Xiaoying Zhang, Chinese-German joint Institute for natural product research / Shaanxi Engineering Research Center for
Tall Gastrodia Tuber and Medical Dogwood / College of Biological Science and Engineering, Shaanxi University of Technology, Hanzhong, China. Tel:
+86-09162641011; Fax: +86-09162641661; E-mail: zhang.xy@nwsuaf.edu.cn

Xiang Liu, Chinese-German joint Institute for natural product research / Shaanxi Engineering Research Center for Tall Gastrodia Tuber and Medical Dogwood / College of Biological Science and Engineering, Shaanxi University of Technology, Hanzhong, China. Tel: +86-09162641011; Fax: +8609162641661; E-mail: zhang@bio.uminho.pt

\begin{abstract}
Background: Vibrio are the main pathogenic bacteria in aquaculture. The flagellin protein C (FlaC) of Vibrio alginolyticus has good immunogenicity and the prospect of potential application in a vaccine.

Objectives: We aimed to evaluate the immunogenicity, protective immunity, and prokaryotic expression fermentation of $V$. alginolyticus $\mathrm{FlaC}$ protein for the vaccine in aquaculture.

Material and Methods: A molecular cloning method was used to construct the expression strain of FlaC protein, and the protein was purified with $\mathrm{Ni}$-affinity chromatography. Polyclonal antiserum was prepared via mice immunized with the FlaC protein. The Western blot and enzyme-linked immunosorbent assay (ELISA) were used to check the specificity and titre of the antiserum. ELISA and pull-down assay detected the interaction between FlaC protein antiserum and Vibrio. The immune protection function of $\mathrm{FlaC}$ protein was detected with mice actively immunized with FlaC protein and challenged by $V$. alginolyticus and $V$. parahaemolyticus. The optimal expression conditions for FlaC protein were detected using an $\mathrm{L}_{9}\left(3^{4}\right)$ orthogonal design model.

Results: The expression strain of $\mathrm{FlaC}$ protein was obtained successfully, and purified FlaC protein was prepared using a mice polyclonal antibody. The $\mathrm{FlaC}$ protein antiserum held a high specificity, and the titre was 1:3200. The antiserum directly interacted with $V$. alginolyticus and $V$. parahaemolyticus, and the FlaC protein demonstrated a significant immune protection function (50\%) against $V$. alginolyticus infection and some immune protection function (41.66\%) against $V$. parahaemolyticus. The optimal expression conditions for FlaC protein included a strain $\mathrm{OD}_{600}$ value of 0.8 , final isopropyl- $\beta$-d-thiogalactoside (IPTG) concentration of $0.1 \mathrm{mmol} / \mathrm{L}$, an inducing time of 8 hours, and an inducing temperature of $28^{\circ} \mathrm{C}$.

Conclusions: This study showed that the FlaC protein possesses a significant immunogenicity and immune protection effect and obtained the optimal fermentation conditions. It is expected to be a potential vaccine against $V$. alginolyticus and V.parahaemolyticus.
\end{abstract}

Keywords: Vibrio alginolyticus; Vibrio parahaemolyticus; FlaJ Protein; Vibrio parahaemolyticus

\section{Background}

Vibrio alginolyticus and Vibrio parahaemolyticus are major aquaculture pathogenic Vibrio (1), 
(2), induces a large number of deaths, and affects the development of aquaculture (3). Pathogens coinfecting humans and marine animals, $V$. alginolyticus and $V$. parahaemolyticus can also cause gastrointestinal diseases and wound infections in humans (4). At present, antibiotics are mainly used in the prevention of animal diseases and the treatment of Vibrio infection (5). This usage inevitably leads to drug residues, bacterial resistance, and environmental pollution (6). Therefore, it is necessary to develop new drug preparations.

Vibrio usually has three kinds of antigens, which are flagellum antigen, bacterium antigen, and surface antigen (7). These antigens provide the interaction between bacteria and host and are a prerequisite for the preparation of vaccine with strong immune protection specificity. Vibrio flagella are essential components in the pathogenic potential of bacteria, because they enhance the motility and adhesion ability of the bacteria (7). They are also important bacterial surface antigens, which are closely related to bacterial immune protection (8). Flagellin protein $\mathrm{C}(\mathrm{FlaC}), \mathrm{FlaB}$, and FlaI of Vibrio can activate the immune protection function of animals, and they have good immunogenicity and hold the prospect of potential application in vaccines. FlaC protein is a granular protein, the main protein responsible for the flagellum fibres of $V$. alginolyticus, providing the basis for the movement of the bacteria (9). It has been found to have good immunogenicity, which activates the immune system of animals and enhances the immune protection function of Lutjanus sanguineus against $V$. alginolyticus (10). Thus, FlaC protein has a good prospect of application in a vaccine against Vibrio infection. It is, therefore, necessary to further research into the immune protection function and fermentation conditions of FlaC protein.

\section{Objectives}

In this study, we aimed to evaluate the immunogenicity, protective immunity, and prokaryotic expression fermentation of $V$. alginolyticus $\mathrm{FlaC}$ protein for the vaccine in aquaculture.

\section{Materials and Methods}

\subsection{Ethics statement}

The animal ethical committee (Ref. no20161120) was approved by the ethics committee of Shaanxi University of Technology, China.

\subsection{Materials}

$V$. alginolyticus, $V$. parahaemolyticus, the Escherichia coli DH5a strain, the E. coli BL21 strain, and pET-32a plasmid were obtained from the Bacterial Conservation Centre, China. Primer synthesis and gene sequencing were completed by the Beijing Oak Science and Technology Corp., China. The mice were obtained from the College of Medicine, Xian Jiaotong University, China.

\subsection{Cloning of FlaC Gene}

The flaC gene primers were designed according to the flaC gene sequence of $V$.alginolyticus (GenBank database accession number FJ617268), including sense primer

5 TAAGGATCCATGGCTGTAACAGTTAGT-3' and anti-sense primer 5'CCTCTCGAGTTACTGCAATAGTGACAT-3', where the first and second underscores represent the sites of the BamH I and Xho I enzyme cleavages, respectively. The $V$. alginolyticus genome was extracted using a genomic extraction kit (TaKaRa, Japan). The polymerase chain reaction (PCR) system consisted of $2.5 \mu \mathrm{L}$ buffer, $2 \mu \mathrm{L}$ dNTP $(10 \mathrm{mmol} / \mathrm{L}), 1.5 \mu \mathrm{L}$ primers $(20 \mu \mathrm{mol} / \mathrm{L}), 4 \mu \mathrm{L}$ template DNA, and $0.2 \mu \mathrm{L}$ Taq enzyme (TaKaRa, Japan). The PCR cycle parameters were pre-denatured for 2 minutes at $94^{\circ} \mathrm{C}$ and were then denatured, annealed, and extended for 32 cycles ( $30 \mathrm{~s}$ at $94^{\circ} \mathrm{C}, 45 \mathrm{~s}$ at $55^{\circ} \mathrm{C}$, and $90 \mathrm{~s}$ at $72^{\circ} \mathrm{C}$ ) and fully extended for 10 minutes at $72^{\circ} \mathrm{C}$. PCR samples were separated and recovered by $0.8 \%$ agarose gel electrophoresis. After the PCR product and $\mathrm{pET}-32 \mathrm{a}$ plasmid vector were digested, the recombinant plasmid pET32a-flaC was obtained by ligase ligation (TaKaRa, Japan). The recombinant plasmid was identified through doubleenzyme digestion and sequencing test. Then, it was transformed into an E. coli BL21 strain to construct the expression strain of FlaC protein.

\subsection{Expression Detection and Purification of FlaC} Protein

The expression and purification were implemented as described above. The flaC gene recombinant strains were cultured overnight and transferred to fresh LB medium. The $\mathrm{OD}_{600}$ value was about 0.5 , and the final concentration of $0.5 \mathrm{mmol} / \mathrm{L}$ isopropyl- $\beta$-Dthiogalactoside (IPTG) was added and cultured at $37^{\circ} \mathrm{C}$ for 5 hours. FlaC protein expression was detected by SDS-PAGE (11). The FlaC strain was loaded using Niaffinity chromatography and purified using the Ni-NTA flow resin method (TaKaRa, Japan).

3.5. Preparation of Mouse Polyclonal Antiserum against FlaC Protein

Kunming mice were randomly selected. The experimental and control groups were immunized with FlaC protein (50 $\mu \mathrm{g}$ per mouse) and phosphate-buffered saline (PBS) solution, respectively. Freund's complete adjuvant (Sigma, USA) was used for the first immunization. The second immunization followed after 14 days, this time using Freund's incomplete adjuvant (Sigma, USA). Seven days later, the third immunization was performed. Then the eyeballs of the mice were 
removed under anaesthesia to obtain $\mathrm{FlaC}$ protein antiserum and stored in a refrigerator at $-80^{\circ} \mathrm{C}$.

\subsection{Specificity and Titre Detection of FlaC Protein Antiserum}

The specificity of $\mathrm{FlaC}$ protein antiserum was determined by the Western blot. Through SDS-PAGE, $V$. alginolyticus protein was transferred to a nitrocellulose (NC) membrane (TaKaRa, Japan). After incubating in skim milk solution for blocking, the membrane was incubated with mouse anti-FlaC protein antiserum and was then added to anti-mouse horseradish antibodies (TaKaRa, Japan). A DAB solution (Sigma, USA) colouration system was used on the NC membrane for band visualization. The FlaC protein serum specificity was determined according to the colour of the NC membrane bands (11).

The titre of the antiserum was detected using the enzyme-linked immunosorbent assay (ELISA). The FlaC protein was dissolved to $0.5 \mu \mathrm{g} / \mu \mathrm{L}$, and $100 \mu \mathrm{L}$ of the solution was added to the 96 -well plate at $37^{\circ} \mathrm{C}$ for 3 hours. After incubating in a skim milk solution for blocking, $100 \mu \mathrm{L}$ of anti-FlaC protein antiserum was added to each well at $37^{\circ} \mathrm{C}$ for 30 minutes. After rinsing, $100 \mu \mathrm{L}$ anti-mouse horseradish antibodies were added to each well. Then, a colouration solution (Sigma, USA) was added to every well at $37^{\circ} \mathrm{C}$ to prevent light from affecting the colour reaction. Finally, a stop solution was added, and a microplate reader (Thermo, USA) was used to detect the $\mathrm{OD}_{450}$ value (11).

3.7. Interaction between FlaC Protein Antiserum, $V$. alginolyticus, and V. parahaemolyticus by ELISA and Pulldown Assay

ELISA and pull-down assay were implemented as described. V.alginolyticus and V. parahaemolyticus were collected at the logarithmic growth phase by centrifuge and washed twice with $\mathrm{NaCl}$ solution $(0.85 \%)$. The bacteria were deactivated and immobilized with oxymethylene at $80^{\circ} \mathrm{C}$ for 90 minutes. Then the samples were dissolved in $0.85 \% \mathrm{NaCl}$ and adjusted to 0.2 at $\mathrm{OD}_{600} \mathrm{~nm}$. Samples of $1 \mathrm{~mL}$ were transferred into 1.5 $\mathrm{mL}$ tubes with $10^{8} \mathrm{CFU}$ bacterial cells. Then $100 \mu \mathrm{L}$ FlaC protein antiserum was added to each tube, with 1.5 $\mu \mathrm{g} / \mu \mathrm{L}$ protein of bovine serum albumin used for the negative control. After rinsing, $100 \mu \mathrm{L}$ anti-mouse horseradish antiserum was also added to each tube. Colouration liquid was added to every tube to prevent a light reaction. After the stop solution was added to each tube, a microplate reader was used to detect the absorbance value at $\mathrm{OD}_{450} \mathrm{~nm}(11)$.

\subsection{Immune Protection Function of FlaC Protein}

Kunming mice were divided between an experimental group and a control group. Purified FlaC protein $(50 \mu \mathrm{g}$ per mouse) was injected three times and the control group was injected with PBS solution. The primary immunization was conducted with Freund's complete adjuvant, and booster doses with Freund's incomplete adjuvant. After the third immunization, the mice were challenged with $V$. alginolyticus and $V$. parahaemolyticus. After 15 days, the relative percentage survival of the mice was measured, and the immune protection rates were expressed as formula of $1-$ (FlaC immunity mortality/non-protein immunity mortality) $\times 100 \%$ (12). The Statistical Package for the Social Science (SPSS) software was used for significance analysis.

\subsection{Induced Expression Condition of FlaC Protein}

The $\mathrm{L}_{9}\left(3^{4}\right)$ orthogonal design model-a four-factor, three-level orthogonal design-was used to detect the optimal expression conditions of $\mathrm{FlaC}$ protein. The factors of the orthogonal design included the strain of $\mathrm{OD}_{600}$ value, IPTG final concentration, inducing time, and inducing temperature, represented as A, B, C, and $D$, respectively (Table 2 ). In accordance with the orthogonal design model, when the $\mathrm{OD}_{600}$ concentration of $\mathrm{FlaC}$ protein expression of the bacteria was reached, corresponding concentrations of IPTG were added to the culture to induce $\mathrm{FlaC}$ protein expression at an appropriate time and temperature. One millilitre of bacterial liquid was harvested and boiled for 5 minutes with $300 \mu \mathrm{L}$ buffer solution. After centrifuge, $10 \mu \mathrm{L}$ samples were added for SDS-PAGE. G-250 dye liquor (Sigma, USA) was used to visualize the FlaC protein bands. Finally, Phoretix 1D software was used to analyse the optical density of the FlaC protein bands, and SPSS software was used to analyse the significance for each factor.

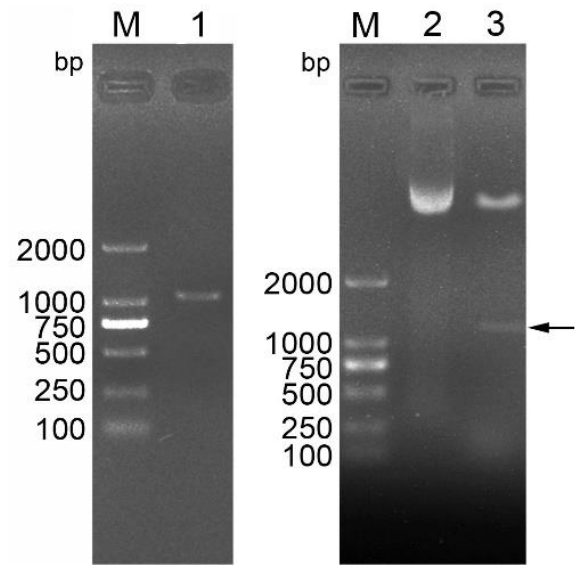

Figure 1. The polymerase chain reaction (PCR) and restriction enzyme analysis of Vibrio alginolyticus flaC gene. M, DNA marker; 1, the PCR of flaC gene; 2 , the recombinant plasmid of flaC gene; 3 , the recombinant plasmid digested by Bam $\mathrm{H}$ I and Xho I. Using PCR, a fragment of about $1155 \mathrm{bp}$ was amplified from the genome of $V$. alginolyticus, which was consistent with the size of the flaC gene. The size of the double-enzyme digestion was consistent with the prediction recombinant gene.

\section{Results}

4.1. Construction of FlaC Gene Prokaryotic Expression Strain

A fragment of about $1155 \mathrm{bp}$ was amplified from the genome of $V$. alginolyticus using PCR; this was 
consistent with the expected size (Fig. 1). The target gene obtained by PCR was linked with pET-32a plasmid. The size of the target gene obtained by the double-enzyme digestion was consistent with the prediction (Fig. 1). Sequencing results also confirmed that the target gene was the same as the flaC gene sequence published by the National Center for Biotechnology Information database. Finally, flaC gene recombinant plasmid was transformed into the E. coli Bl21 strain to obtain the expression strain of FlaC protein.

\subsection{Expression Detection and Purification of FlaC} Protein

To verify the expression of the recombinant target protein, the bacteria containing the recombinant plasmid were induced with IPTG, and a protein band with a molecular weight of $\sim 62 \mathrm{kDa}$ was obtained, containing $\sim 42.35 \mathrm{kDa}$ of FlaC protein and $20.4 \mathrm{kDa}$ fusion protein of $\mathrm{pET}-32 \mathrm{a}$ plasmid, which was consistent with the expected weight (Fig. 2). The purified $\mathrm{FlaC}$ protein was obtained using Ni-affinity chromatography (Fig. 2).

\subsection{Specificity and Titre of FlaC Protein Antiserum}

The Western blot was used to detect the specificity of the $\mathrm{FlaC}$ mice antiserum, and it detected a single band of $\mathrm{FlaC}$ protein, indicating that the $\mathrm{FlaC}$ protein antiserum had good specificity (Fig. 3A). The antiserum titre of $\mathrm{FlaC}$ protein reached 1:3200 with ELISA (Fig. 3B).

\subsection{Interaction between FlaC Protein Antiserum, V.} alginolyticus, and $V$. parahaemolyticus

The interaction between $\mathrm{FlaC}$ protein antiserum and Vibrio was detected using ELISA and pull-down assay. There was interaction between $\mathrm{FlaC}$ protein antiserum and $V$. alginolyticus until the 1:500 serum titre, and FlaC protein antiserum and $V$. parahaemolyticus until the 1:400 serum titre (Fig. 4). FlaC protein antiserum and major aquaculture pathogenic Vibrio formed antigenantibody complexes, which probably provided for antigen presentation. It was revealed that $\mathrm{FlaC}$ protein may have a preferable immunogenicity.

\subsection{Immunological Protection of FlaC Protein}

Mice were immunized with FlaC protein and challenged with $V$. alginolyticus and $V$. parahaemolyticus to detect the immunological protection of FlaC protein. The mice developed severe toxic symptoms, such as fluffy folds, sluggish activity, listlessness, and lethargy, and a large number died within 48 hours. After 4 days, death was controlled, and the mice gradually recovered. The FlaC protein demonstrated a significant immune protection function (50\%) against $V$. alginolyticus infection and some immune protection function (41.66\%) against $V$. parahaemolyticus (Table 1).

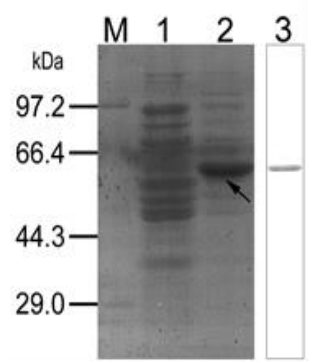

Figure 2. Expression detection and protein purification of flagellin protein $\mathrm{C}(\mathrm{FlaC}) . \mathrm{M}$, protein marker; 1 , no inducing with isopropyl- $\beta$ D-thiogalactoside (IPTG); 2 , inducing with IPTG; 3 , purification of FlaC protein. The recombinant protein bacteria were induced with IPTG, obtaining a protein band with a molecular weight of $\sim 62 \mathrm{kDa}$, containing $\sim 42.35 \mathrm{kDa}$ of FlaC protein and $20.4 \mathrm{kDa}$ of fusion protein, which was consistent with the expected weight. The purified FlaC protein was obtained with $\mathrm{Ni}$-affinity chromatography and showed one band.

\subsection{Optimization of Prokaryotic Expression Conditions of} FlaC Protein

In order to detect FlaC protein expression, orthogonal design experiments were conducted. The expression map of FlaC protein was obtained by SDS-PAGE, which showed that the FlaC protein expression amount differed under different induction conditions (Fig. 5). The optical density value of the FlaC protein band was obtained by Phoretix 1D software, and range analysis was conducted (Table 2). Comparing K1, K2, and $\mathrm{K} 3$, the optimal expression condition of FlaC protein was $\mathrm{A} 2, \mathrm{~B} 1, \mathrm{C} 2$, and $\mathrm{D} 1$, which meant a strain $\mathrm{OD}_{600}$ value of 0.8 , a final IPTG concentration of 0.1 $\mathrm{mmol} / \mathrm{L}$, an induction time of 8 hours, and an induction temperature of $28^{\circ} \mathrm{C}$. The variance analysis of the optical density data showed that four factors reached significance, including the strain $\mathrm{OD}_{600}$ value, final IPTG concentration, induction time, and induction temperature (Table 3).
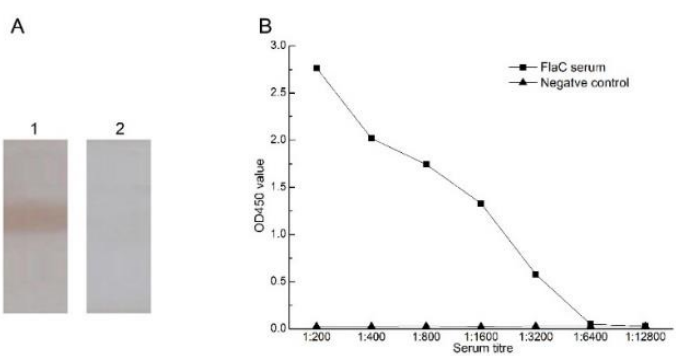

Figure 3. Specificity and titre of flagellin protein $\mathrm{C}(\mathrm{FlaC})$ antiserum. A and $\mathrm{B}$ show the specificity and titre, respectively, of the FlaC protein antiserum. 1 and 2 show the FlaC protein antiserum and negative control, respectively. 1 shows that one band was visualized, indicating that the FlaC protein antiserum had good specificity. As the $\mathrm{FlaC}$ protein antiserum titre increased, the $\mathrm{OD}_{450}$ value decreased; the FlaC protein antiserum titre was $1: 3200$. 

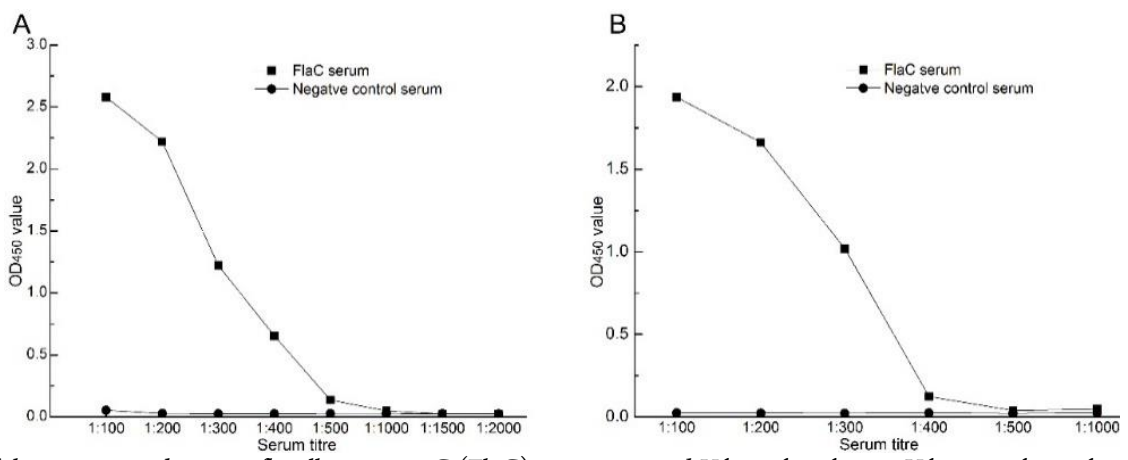

Figure 4. Analysis of the interaction between flagellin protein $\mathrm{C}(\mathrm{FlaC})$ antiserum, and Vibrio alginolyticus, Vibrio parahaemolyticus. A and B show the interaction between $\mathrm{FlaC}$ protein antiserum and $V$. alginolyticus, $V$. parahaemolyticus. As the $\mathrm{FlaC}$ protein antiserum titre increased, the $O D_{450}$ value decreased. The interaction between $\mathrm{FlaC}$ protein antiserum and $V$. alginolyticus can be found until the 1:500 serum titre, and FlaC protein antiserum and V. parahaemolyticus until the 1:400 serum titre.

Table 1. Active Immunity FlaC and Challenging with Vibrio alginolyticus and Vibrio parahaemolyticus in Mice

\begin{tabular}{lcccccccc}
\hline Bacterial Name & \multicolumn{3}{c}{ FlaC } & \multicolumn{3}{c}{ Control } \\
\hline & Nos & Alive & ADR $(\%)$ & RPS $(\%)$ & Nos & Alive & ADR (\%) & RPS (\%) \\
V. alginolyticus & 15 & 9 & 40 & $50^{*}$ & 15 & 3 & 80 & - \\
V.parahaemolyticus & 15 & 8 & 46.67 & 41.66 & 15 & 3 & 80 & - \\
\hline ADD & 15 &
\end{tabular}

ADR, accumulating death rates; FlaC, flagellin protein C; RPS, relative percent survivals

RPS $(\%)=1-($ FlaC immunity mortality/non-protein immunity mortality $) \times 100 \%$.

${ }^{*} \mathrm{P}<0.05$ (compared to the control group which received phosphate-buffered saline only)

A

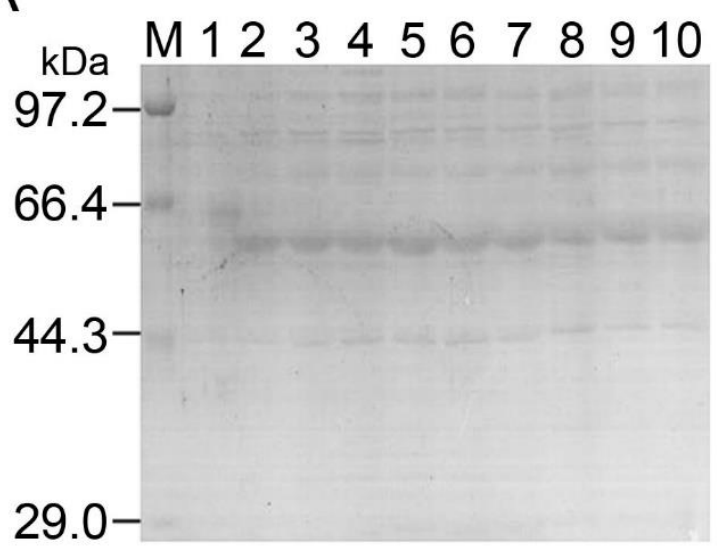

B

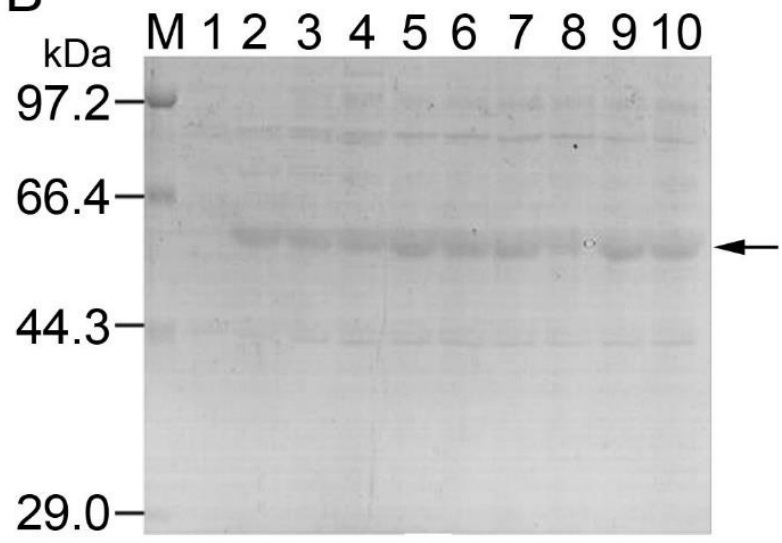

C

$\mathrm{kDa}$

M12345678910

97.2-

66.4-

44.3

29.0

Figure 5. The orthogonal design experiment of the expression strain of flagellin protein $\mathrm{C}(\mathrm{FlaC})$ induced by isopropyl- $\beta$-D-thiogalactoside (IPTG). A, $\mathrm{B}$, and $\mathrm{C}$ represent three repeated experiments. $\mathrm{M}$, protein marker; 1 , no induction strain. The 2,3 , and 4 mean a strain $\mathrm{OD}_{600}$ value of 0.5 ; IPTG final concentration of $0.1,0.3$, and $0.5 \mathrm{mmol} / \mathrm{L}$; induction temperature of $28^{\circ} \mathrm{C}, 32^{\circ} \mathrm{C}$, and $37^{\circ} \mathrm{C}$; and induction time of 3,8 , and 12 hours. The 5,6 , and 7 mean a strain $\mathrm{OD}_{600}$ value of 0.8 ; IPTG final concentration of $0.1,0.3$, and $0.5 \mathrm{mmol} / \mathrm{L}$; induction temperature of $32^{\circ} \mathrm{C}, 28^{\circ} \mathrm{C}$, and $37^{\circ} \mathrm{C}$; and induction time of 8,12 , and 3 hours. The 8,9 , and 10 mean a strain $\mathrm{OD}_{600}$ value of 1.0 ; final IPTG concentration of $0.1,0.3$, and $0.5 \mathrm{mmol} / \mathrm{L}$; induction temperature of $32^{\circ} \mathrm{C}, 37^{\circ} \mathrm{C}$, and $28^{\circ} \mathrm{C}$; and induction time of 12,3 , and 8 hours. 


\section{Discussion}

The FlaC protein has good immunogenicity, can stimulate an immune response, and has a potential application in a vaccine (10). In this study, the FlaC protein was obtained by molecular cloning and purification, and the mouse polyclonal antiserum was prepared with good specificity. The titre of FlaC protein antiserum reached 1:3200. Compared with monoclonal antibodies, polyclonal antibodies are more convenient and economical to prepare $(13,14)$ and are widely used in the immune protection function analysis of the protein (15). This study obtained the FlaC protein antiserum, which lays the foundation for the immunological function of FlaC protein.

Our research showed that there was interaction between $\mathrm{FlaC}$ protein antiserum and major aquaculture pathogenic Vibrio of $V$. alginolyticus and $V$. parahaemolyticus; they formed antigenantibody complexes, which probably provided for antigen presentation $(16,17)$. Therefore, the body's immune system can easily identify Vibrio with FlaC protein antiserum mediation and eliminate pathogenic bacteria. Thus, the FlaC protein was expected to display good immunogenicity. Some experiments have found that the $\mathrm{FlaC}$ protein provided immune protection for $L$. sanguineus against $V$. alginolyticus infection $(10,18)$. This study showed that the FlaC protein had a significant immune protection function (50\%) against $V$. alginolyticus infection and some immune protection function $(41.66 \%)$ against $V$. parahaemolyticus, and it laid a theoretical foundation for developing $\mathrm{FlaC}$ immunization agents.

\begin{tabular}{|c|c|c|c|c|c|}
\hline No. & $\mathbf{A}$ & B ( $\mathrm{mmol} / \mathrm{L})$ & C (h) & $\mathbf{D}\left({ }^{\circ} \mathrm{C}\right)$ & Optical Density/SD $\left(\times 10^{4}\right)$ \\
\hline $\mathbf{1}$ & 0.5 & 0.1 & 3 & 28 & $4.708 \pm 0.393$ \\
\hline 2 & 0.5 & 0.3 & 8 & 32 & $5.957 \pm 0.242$ \\
\hline 3 & 0.5 & 0.5 & 12 & 37 & $7.310 \pm 0.070$ \\
\hline 4 & 0.8 & 0.1 & 8 & 37 & $7.534 \pm 0.299$ \\
\hline 5 & 0.8 & 0.3 & 12 & 28 & $6.311 \pm 0.579$ \\
\hline 6 & 0.8 & 0.5 & 3 & 32 & $5.220 \pm 0.215$ \\
\hline 7 & 1.0 & 0.1 & 12 & 32 & $5.575 \pm 0.292$ \\
\hline 8 & 1.0 & 0.3 & 3 & 37 & $5.726 \pm 0.241$ \\
\hline 9 & 1.0 & 0.5 & 8 & 28 & $4.016 \pm 0.260$ \\
\hline K1 (mean value 1) & 5.992 & 5.999 & 5.518 & 6.012 & \\
\hline K2 (mean value 2$)$ & 6.355 & 5.998 & 6.527 & 5.584 & \\
\hline K3 (mean value 3 ) & 5.107 & 5.515 & 6.399 & 5.859 & \\
\hline Range analysis & 1.249 & 0.484 & 1.009 & 0.428 & \\
\hline
\end{tabular}

$\mathrm{A}, \mathrm{B}, \mathrm{C}$, and D mean the induction strain $\mathrm{OD}_{600}$ value, induction of IPTG concentration, induction time, and induction temperature, respectively. Compared to the analysis size of the mean value of $\mathrm{K} 1, \mathrm{~K} 2$, and $\mathrm{K} 3$, the optimal inducing expression condition of FlaC protein was $\mathrm{A} 2$, B1, $\mathrm{C} 2$, and $\mathrm{D} 1$, respectively.

In order to obtain the $\mathrm{FlaC}$ protein by large-scale fermentation, different factors and levels were set, using an $\mathrm{L}_{9}\left(3^{4}\right)$ orthogonal design model (19). We found that the optimal induction conditions of $\mathrm{FlaC}$ protein were a strain $\mathrm{OD}_{600}$ value of 0.8 , final IPTG concentration of $0.1 \mathrm{mmol} / \mathrm{L}$, an induction time of 8 hours, and an induction temperature of $28^{\circ} \mathrm{C}$. IPTG is an active inducer of $\beta$-galactosidase, and a high concentration of IPTG has some cytotoxicity, inhibiting protein expression $(20,21)$; this fact is consistent with how our research showed that the low concentration of IPTG $(0.1 \mathrm{mmol} / \mathrm{L})$ improved $\mathrm{FlaC}$ protein expression. Some researchers have found that the protein expression was inhibited when induced for a long time $(22,23)$, and our research showed the same. A suitable induction time is favourable to the protein expression, and the cost of raw materials is saved compared to using a blind setting for induction time (24). To further verify this conclusion, our research showed that the 8 -hour induction time was the best inducement condition. Thus, we suggest that the actual fermentation production would be best with strain induction at a logarithmic growth period, with a low concentration of IPTG, at a suitable induction time, and at a low temperature.

Table 3. Variance results of FlaC protein expression

\begin{tabular}{lccc}
\hline Factor & \multicolumn{3}{c}{ Optical Density Value Analysis } \\
\hline & Mean Square & $\boldsymbol{F}$-value & $\boldsymbol{P}$-value \\
A & 3.718 & 37.191 & 0 \\
B & 0.624 & 6.237 & 0.009 \\
C & 3.139 & 31.392 & 0 \\
D & 8.027 & 80.284 & 0 \\
\hline A
\end{tabular}

$\mathrm{A}, \mathrm{B}, \mathrm{C}$, and D mean strain OD600 value, IPTG concentration, induction time, and induction temperature, respectively. ${ }^{*} \mathrm{P}<0.05$ means the factor has a significant difference from the control group.

\section{Conclusions}

The present study obtained the expression strain of $V$. alginolyticus $\mathrm{FlaC}$ protein, and the optimal fermentation conditions were determined. The FlaC protein was purified and antiserum was prepared, and it was confirmed that the $\mathrm{FlaC}$ protein possesses a preferable immunogenicity and excellent immune protection effect on $V$. alginolyticus and $V$. parahaemolyticus infection. Our findings would provide a scientific basis 
for the vaccine development on major aquaculture pathogenic Vibrio.

\section{Conflict of Interest}

The authors declare no conflict of interest.

\section{Acknowledgements}

This research was supported by the High-end Foreign Experts Recruitment Program of State Administration of Foreign Experts Affairs (No. GDT20176100048 and No. GDT20186100426), the Scientific Research Program of the Shaanxi Provincial Education Department (No. 17JK0137), and the Project of Shaanxi University of Technology (No. SLGQD1803).

\section{References}

1. Baker-Austin C, Oliver JD, Alam M, Ali A, Waldor MK, Qadri F, et al. Vibrio spp. infections. Nat Rev Dis Primers. 2018;4(1):8. doi: 10.1038/s41572-018-00058 pmid: 30002421

2. Zhu F, Qian X, Wang Z. Molecular characterization of minichromosome maintenance protein (MCM7) in Scylla paramamosain and its role in white spot syndrome virus and Vibrio alginolyticus infection. Fish Shellfish Immunol. 2018;83:104-114. doi: 10.1016/j.fsi.2018.09.028 pmid: 30205202

3. Hamza F, Kumar AR, Zinjarde S. Efficacy of cell free supernatant from Bacillus licheniformis in protecting Artemia salina against Vibrio alginolyticus and Pseudomonas gessardii. Microb Pathog. 2018;116:335344. doi: 10.1016/j.micpath.2018.02.003 pmid: 29408316

4. Zidour M, Chevalier M, Belguesmia Y, Cudennec B, Grard T, Drider D, et al. Isolation and Characterization of Bacteria Colonizing Acartia tonsa Copepod Eggs and Displaying Antagonist Effects against Vibrio anguillarum, Vibrio alginolyticus and Other Pathogenic Strains. Front Microbiol. 2017;8:1919. doi: 10.3389/fmicb.2017.01919 pmid: 29085344

5. Mechri B, Monastiri A, Medhioub A, Medhioub MN, Aouni M. Molecular characterization and phylogenetic analysis of highly pathogenic Vibrio alginolyticus strains isolated during mortality outbreaks in cultured Ruditapes decussatus juvenile. Microb Pathog. 2017;111:487-496. doi: 10.1016/j.micpath.2017.09.0 20 pmid: 28923608

6. Zouiten A, Mehri I, Beltifa A, Ghorbel A, Sire O, Van Loco J, et al. Designation of pathogenic resistant bacteria in the Sparusaurata sea collected in Tunisia coastlines: Correlation with high performance liquid chromatography-tandem mass spectrometry analysis of antibiotics. Microb Pathog. 2017;106:3-8. doi: 10.1016/j.micpath.2017.01.004 pmid: 28062288

7. Dongre M, Singh B, Aung KM, Larsson P, Miftakhova $\mathrm{R}$, Persson $\mathrm{K}$, et al. Flagella-mediated secretion of a novel Vibrio cholerae cytotoxin affecting both vertebrate and invertebrate hosts. Commun Biol. 2018;1:59. doi: 10.1038/s42003-018-0065-z pmid: 30271941

8. Echazarreta MA, Kepple JL, Yen LH, Chen Y, Klose KE. A Critical Region in the FlaA Flagellin Facilitates Filament Formation of the Vibrio cholerae Flagellum. J
Bacteriol. 2018;200(15). doi: 10.1128/JB.00029-18 pmid: 29581407

9. Xing J, Zhou X, Tang X, Sheng X, Zhan W. FlaC supplemented with VAA, OmpK or OmpR as bivalent subunit vaccine candidates induce immune responses against Vibrio anguillarum in flounder (Paralichthys olivaceus). Vaccine. 2018;36(10):1316-1322. doi: 10.1016/j.vaccine.2017.11.074 pmid: 29397223

10. Liang H, Xia L, Wu Z, Jian J, Lu Y. Expression, characterization and immunogenicity of flagellin $\mathrm{FlaC}$ from Vibrio alginolyticus strain HY9901. Fish Shellfish Immunol. 2010;29(2):343-348. doi: 10.1016/j.fsi.2010.04.003 pmid: 20420917

11. Liu X, Chen C, Chen C, Ding R, Marslin G. Construction of a Recombinant OmpC Dominant Epitope-Based Vaccine Against Escherichia coli and Evaluation of Its Immunogenicity and Protective Immunity. Jundishapur J Microbiol. 2017;10(11). doi: 10.5812/jjm.55652

12. Liu X, Yang MJ, Wang SN, Xu D, Li H, Peng XX. Differential Antibody Responses to Outer Membrane Proteins Contribute to Differential Immune Protections between Live and Inactivated Vibrio parahemolyticus. J Proteome Res. 2018;17(9):29872994. doi: 10.1021/acs.jproteome.8b00176 pmid: 30095909

13. Zhang C, Huang X, Chen S, Li Y, Li Y, Wang X, et al. Epitope clustering analysis for vaccine-induced human antibodies in relationship to a panel of murine monoclonal antibodies against HPV16 viral capsid. Vaccine. 2018;36(45):6761-6771. doi: 10.1016/j.vaccine.2018.09.035 pmid: 30287156

14. Franka R, Carson WC, Ellison JA, Taylor ST, Smith TG, Kuzmina NA, et al. In Vivo Efficacy of a Cocktail of Human Monoclonal Antibodies (CL184) Against Diverse North American Bat Rabies Virus Variants. Trop Med Infect Dis. 2017;2(3). doi: 10.3390/tropicalmed2030048 pmid: 30270905

15. Ferlito C, Biselli R, Mariotti S, von Hunolstein C, Teloni R, Ralli L, et al. Tetanus-diphtheria vaccination in adults: the long-term persistence of antibodies is not dependent on polyclonal B-cell activation and the defective response to diphtheria toxoid re-vaccination is associated to HLADRB1 ${ }^{*} 01$. Vaccine. 2018;36(45):6718-6725. doi: 10.1016/j.vaccine.201 8.09.041 pmid: 30269918

16. Junker F, Krishnarajah S, Qureshi O, Humphreys D, Fallah-Arani F. A simple method for measuring immune complex-mediated, Fc gamma receptor dependent antigen-specific activation of primary human T cells. $J$ Immunol Methods. 2018;454:32-39. doi: 10.1016/j.jim.2017.12.002 pmid: 29258749

17. Rolle A, Meyer M, Calderazzo S, Jager D, Momburg F. Distinct HLA-E Peptide Complexes Modify AntibodyDriven Effector Functions of Adaptive NK Cells. Cell Rep. 2018;24(8):1967-1976 e1964. doi: 10.1016/j.celrep.2018.07.069 pmid: 30134159

18. Razavi SS, Peyvandi H, Badrkhani Jam AR, Safari F, Teymourian H, Mohajerani SA. Magnesium Versus Bupivacaine Infiltration in Controlling Postoperative Pain in Inguinal Hernia Repair. Anesth Pain Med. 2015;5(6):e30643. doi: 10.5812/aapm.30643 pmid: 26705525 
19. Liu X, Chen C, Chen C, Marslin G, Ding R, Wu S. Construction and evaluation of a novel triple cell epitopebased polypeptide vaccine against cow mastitis induced by Staphylococcus aureus, Escherichia coli and Streptococcus. Trop J Pharm Res. 2017;16(10):24772486.

20. Ghaderi H, Arasteh J, Hesampour A. Using response surface methodology in combination with PlackettBurman design for optimization of culture media and extracellular expression of Trichoderma reesei synthetic endoglucanase II in Escherichia coli. Mol Biol Rep. 2018;45(5):1197-1208. doi: 10.1007/s11033-0184272-y pmid: 30032381

21. Khademi F, Yousefi-Avarvand A, Derakhshan M, Meshkat Z, Tafaghodi M, Ghazvini K. Mycobacterium tuberculosis $\mathrm{HspX} / \mathrm{EsxS}$ fusion protein: gene cloning, protein expression, and purification in Escherichia coli. Rep Biochem Mol Biology. 2017;6(1):15-21.
22. Lotfi H, Hejazi MA, Heshmati MK, Mohammadi SA, Zarghami N. Optimizing expression of antiviral cyanovirin- $\mathrm{N}$ homology gene using response surface methodology and protein structure prediction. Cell Mol Biol (Noisy-le-grand). 2017;63(9):96-105. doi: 10.14715/cmb/2017.63.9.17 pmid: 28980928

23. Mesgari-Shadi A, Sarrafzadeh MH, Barar J, Omidi Y. Cost-effective batch production process of $\mathrm{scFv}$ antibody in Escherichia coli. Hum Antibodies. 2017;26(3):149-157. doi: 10.3233/HAB-180333 pmid: 29526846

24. van Rijswijck IMH, van Mastrigt O, Pijffers G, WolkersRooijackers JCM, Abee T, Zwietering $\mathrm{MH}$, et al. Dynamic modelling of brewers' yeast and Cyberlindnera fabianii co-culture behaviour for steering fermentation performance. Food Microbiol. 2019;83:113-121. doi: 10.1016/j.fm.2019.04.010 pmid: 31202402 